\title{
Article
}

\section{Relation between Serum Creatine Phosphokinase Levels and Acute Kidney Injury among ST-Segment Elevation Myocardial Infarction Patients}

\author{
David Zahler ${ }^{1,2, *(\mathbb{D}}$, Keren-Lee Rozenfeld ${ }^{1}$, Ilan Merdler ${ }^{1}{ }^{(\mathbb{D}}$, Tamar Itach ${ }^{2}$, Samuel Morgan ${ }^{1}{ }^{(}$, Dana Levit ${ }^{2}$, \\ Shmuel Banai ${ }^{1}$ and Yacov Shacham ${ }^{1}$ \\ 1 Department of Cardiology, Tel-Aviv Sourasky Medical Center Affiliated to the Sackler Faculty of Medicine, \\ Tel-Aviv University, Tel-Aviv 64239, Israel; kerenlees@gmail.com (K.-L.R.); ilanmerdler@gmail.com (I.M.); \\ samuelmorgan@mail.tau.ac.il (S.M.); shmuelb@tlvmc.gov.il (S.B.); kobys@tlvmc.gov.il (Y.S.) \\ 2 Internal Medicine Department H, Tel-Aviv Sourasky Medical Center Affiliated to the Sackler Faculty of \\ Medicine, Tel-Aviv University, Tel-Aviv 64239, Israel; itachtamar@gmail.com (T.I.); \\ levit.dana@gmail.com (D.L.) \\ * Correspondence: david.zahler@gmail.com; Tel.: +972-3-6973222 or +972-52-7006041; Fax: +972-3-6973704
}

Citation: Zahler, D.; Rozenfeld, K.-L.; Merdler, I.; Itach, T.; Morgan, S.; Levit, D.; Banai, S.; Shacham, Y. Relation between Serum Creatine Phosphokinase Levels and Acute Kidney Injury among ST-Segment Elevation Myocardial Infarction Patients. J. Clin. Med. 2022, 11, 1137. https://doi.org/10.3390/ jcm11041137

Academic Editor: Massimo Mancone

Received: 12 January 2022 Accepted: 15 February 2022 Published: 21 February 2022

Publisher's Note: MDPI stays neutral with regard to jurisdictional claims in published maps and institutional affiliations.

Copyright: () 2022 by the authors Licensee MDPI, Basel, Switzerland. This article is an open access article distributed under the terms and conditions of the Creative Commons Attribution (CC BY) license (https:// creativecommons.org/licenses/by/ $4.0 /)$.

\begin{abstract}
Background: Among patients with rhabdomyolysis, the leakage of intracellular skeletal muscle content such as creatine phosphokinase (CPK) into the bloodstream has been associated with an increased risk of acute kidney injury (AKI). We evaluated the possible relationship between serum CPK levels and AKI occurrence among patients with myocyte injury secondary to ST-elevation myocardial infarction (STEMI). Methods: We retrospectively included 2794 patients with STEMI. Patients were stratified according to peak serum CPK levels into mild ( $<1000 \mathrm{U} / \mathrm{L}, n=1603)$, moderate (1000-5000 U/L, $n=1111)$, and severe ( $>5000 \mathrm{U} / \mathrm{L}, n=80)$ categories. The occurrence of AKI was defined by the KDIGO criteria as an increase in serum creatinine (sCR) $\geq 0.3 \mathrm{mg} / \mathrm{dL}$ within $48 \mathrm{~h}$ following PCI. The predictive value of CPK for the risk of AKI occurrence was assessed using multivariate logistic regression models. Results: The overall occurrence of AKI was $10.4 \%$. Incidence of AKI showed a gradual increase between patients with mild, moderate, and severe serum CPK level elevations $(7.8 \%$ vs. $11 \%$ vs. $26 \%$ respectively; $p<0.001)$. In multivariate logistic regression models, both moderate or higher (OR 1.6, 95\% CI 1.1-2.2; $p=0.01$ ) and severe (OR 2.8 95\% CI 1.4-5.6; $p=0.004$ ) serum CPK level elevations were independently associated with AKI. Conclusions: Among STEMI patients, elevated CPK levels were associated with AKI. This association is presumably independent; however, it remains unclear whether it is due to direct toxic (myoglobin-related) or hemodynamic effects (poor left ventricular function). Further studies are required to reveal the underlying mechanism.
\end{abstract}

Keywords: ST-segment elevation myocardial infarction; acute myocardial infarction; creatine phosphokinase; acute kidney injury

\section{Introduction}

Severe rhabdomyolysis (RM) is often complicated by acute kidney injury (AKI) [1]. Muscular cell wall disruption causes the release of intracellular elements such as myoglobin and creatine phosphokinase (CPK) [2]. Although not directly implicated in the pathogenesis of AKI during RM, CPK is routinely used as a serum biomarker for skeletal muscle damage and correlates with the risk of renal injury [3]. Creatine phosphokinase myocardial band (CPK-MB), an isoenzyme of CPK, is a well-established marker of myocardial cell damage [4]. At the time of acute myocardial infarction (AMI), increases in both CPK-MB and total CPK levels are observed [5].

AKI following AMI is common and associated with adverse outcomes [6,7]. However, as opposed to skeletal muscle disruption, the possible predictive significance of CPK as 
a marker for impending AKI in acute myocardial damage has not yet been investigated. We aimed to evaluate the possible utility of CPK as a marker for AKI occurrence among ST-elevation myocardial infarction (STEMI) patients.

\section{Material and Methods}

We performed a retrospective, single-center observational study at the Tel-Aviv Sourasky Medical Center, a tertiary referral hospital with a 24/7 primary PCI service. We included 2958 consecutive patients admitted between October 2007 and December 2019 to the cardiac intensive care unit (CICU) with a diagnosis of acute STEMI. Patients treated either conservatively or by thrombolysis were excluded $(n=31)$, as well as 63 patients whose diagnosis on discharge was a condition other than STEMI (e.g., myocarditis or takotsubo cardiomyopathy). In addition, we excluded patients who died within $24 \mathrm{~h}$ of admission $(n=45)$, presuming insufficient time for AKI to occur. Patients requiring chronic dialysis treatment $(n=12)$ or patients with no documented serum CPK levels $(n=13)$ were excluded as well.

The final study population included 2794 patients whose baseline demographics, cardiovascular history, clinical risk factors, treatment characteristics, and laboratory results were all retrieved from hospital electronic medical records. Diagnosis of STEMI was established in accordance with published guidelines including a typical chest pain history, diagnostic electrocardiographic changes, and serial elevation of cardiac biomarkers [8]. Coronary angioplasty was performed on patients with symptoms $\leq 12 \mathrm{~h}$ in duration as well as in patients with symptoms lasting $12-24 \mathrm{~h}$ in duration if the symptoms persisted at the time of admission.

\subsection{Laboratory Data}

Serum CPK levels were measured upon CICU admission and at least once daily until discharge. Peak CPK levels were defined as the highest level obtained throughout hospitalization. Although there is no existing unified definition, patients were stratified into three groups based on peak serum CPK levels: mild (<1000 U/L), moderate (1000-5000 U/L), and severe ( $>5000 \mathrm{U} / \mathrm{L})$, as suggested in previous reports [9-11].

The serum creatinine $(\mathrm{sCr})$ level was determined at hospital admission, prior to primary PCI and at least daily throughout the CICU stay, and was available for all analyzed patients. Estimated glomerular filtration rate (eGFR) was determined using the Chronic Kidney Disease Epidemiology Collaboration (CKD-EPI) formula [12].

Admission eGFR of $\leq 60 \mathrm{~mL} / \mathrm{min} / 1.73 \mathrm{~m}^{2}$ defined patients with chronic kidney disease (CKD) [13]. AKI was diagnosed by the Kidney Disease Improving Global Outcomes (KDIGO) criteria and defined as an $\mathrm{sCr}$ rise $\geq 0.3 \mathrm{mg} / \mathrm{dL}$ within $48 \mathrm{~h}$ following CICU admission, compared with admission levels [13]. In a subset of 224 patients admitted to the CICU between February 2019 and December 2019, venous blood was drawn 24 h following CICU admission in order to evaluate plasma neutrophil gelatinase-associated lipocalin (NGAL) levels. NGAL levels were analyzed using NGAL rapid ELISA kits (Bioporto Diagnostics, Copenhagen, Denmark).

\subsection{Statistical Analysis}

Continuous variables were presented as mean and standard deviation and compared with one-way analysis of variance (ANOVA) when normally distributed. Median and interquartile range were used in non-normally distributed variables. These variables were compared with the Kruskal-Wallis $\mathrm{H}$ test. Categorical variables are presented as percentages, and $p$ values were calculated with the chi-square test. Independent predictors of AKI were determined using multivariate binary logistic regression models adjusted for all baseline variables found to be significant in univariate analysis. A two-tailed $p$ value of $<0.05$ was considered significant for all analyses. All analyses were performed with the SPSS software (SPSS Inc., Chicago, IL, USA). 


\section{Results}

A total of 2794 patients were included ( $81 \%$ males, mean age of $62 \pm 13$ ), of whom 1603 (57\%) had mild, 1111 (40\%) had moderate, and 80 (3\%) had severe peak CPK level elevations. The median time for peak CPK measurement was 19 (IQR 12-28) hours. Baseline demographic and clinical characteristics for the study population stratified by CPK levels are presented in Table 1. Patients with higher CPK levels were more likely to be younger men and to have fewer comorbidities. In addition, patients with higher CPK levels showed lower left ventricular ejection fraction and higher peak troponin levels.

Table 1. Baseline characteristics.

\begin{tabular}{|c|c|c|c|c|}
\hline & $\begin{array}{c}\text { Mild } \\
(\mathrm{CPK}<1000 \mathrm{U} / \mathrm{L}) \\
n=1603\end{array}$ & $\begin{array}{c}\text { Moderate } \\
(\mathrm{CPK}=1000-5000 \mathrm{U} / \mathrm{L}) \\
n=1111\end{array}$ & $\begin{array}{c}\text { Severe } \\
\begin{array}{c}(\mathrm{CPK}>5000 \mathrm{U} / \mathrm{L}) \\
n=80\end{array}\end{array}$ & $p$ Value \\
\hline Age (years), mean $\pm \mathrm{SD}$ & $63 \pm 13$ & $61 \pm 13$ & $59 \pm 13$ & $<0.001$ \\
\hline Gender (male), $n(\%)$ & $1273(79)$ & $928(84)$ & $72(90)$ & 0.003 \\
\hline Hypertension, $n(\%)$ & $773(48)$ & $447(40)$ & $28(35)$ & $<0.001$ \\
\hline Diabetes mellitus, $n(\%)$ & $440(27)$ & $221(20)$ & $10(13)$ & $<0.001$ \\
\hline Family history of CAD, $n(\%)$ & $340(21)$ & $252(23)$ & $21(26)$ & 0.42 \\
\hline Past AMI, $n(\%)$ & $264(17)$ & $134(12)$ & $5(6)$ & $<0.001$ \\
\hline Smoking, $n(\%)$ & $767(48)$ & $593(53)$ & $37(46)$ & 0.01 \\
\hline Hyperlipidemia, $n(\%)$ & $833(52)$ & $517(47)$ & $24(30)$ & $<0.001$ \\
\hline Peak troponin $(\mathrm{ng} / \mathrm{L})$, mean $\pm \mathrm{SD}$ & $8938 \pm 31,958$ & $37,911 \pm 108,427$ & $70,779 \pm 152,259$ & $<0.001$ \\
\hline $\operatorname{LVEF}(\%)$, mean \pm SD & $48 \pm 8$ & $45 \pm 7$ & $40 \pm 8$ & $<0.001$ \\
\hline $\mathrm{LVEF} \leq 45 \%, n(\%)$ & $651(41)$ & $683(63)$ & $70(88)$ & $<0.001$ \\
\hline
\end{tabular}

CPK, creatine phosphokinase; SD, standard deviation; CAD, coronary artery disease; AMI, acute myocardial infarction; LVEF, left ventricular ejection fraction.

Table 2 presents the kidney-related baseline and outcome data. Patients with higher CPK level elevations were more likely to have chronic kidney disease. There was a gradual increase in the occurrence of AKI among the CPK groups (mild 7.8\%, moderate $11 \%$, severe $26 \%, p<0.001$ ). Similar increases were observed in $\mathrm{sCr}$ change, peak $\mathrm{sCr}$, and $24 \mathrm{~h}$ plasma NGAL levels (in a subset of 224 patients in whom NGAL levels were available). More significant CPK level elevations correlated with rising $\mathrm{sCr}(p<0.001$, Figure 1$)$. In two multivariate binary logistic regression models including all variables found to be predictive in univariate analysis, CPK levels were independently associated with a higher risk of AKI (CPK $\geq 1000$ (OR 1.6, 95\% CI 1.1-2.2; $p=0.01$ ) and CPK $\geq 5000$ (OR 2.8, 95\% CI 1.4-5.6; $p=0.004)$, Table 3).

Table 2. Baseline and in-hospital renal outcomes.

\begin{tabular}{|c|c|c|c|c|}
\hline & $\begin{array}{c}\text { Mild } \\
(\mathrm{CPK}<1000 \mathrm{U} / \mathrm{L}) \\
n=1603\end{array}$ & $\begin{array}{c}\text { Moderate } \\
(\mathrm{CPK}=1000-5000 \mathrm{U} / \mathrm{L}) \\
n=1111\end{array}$ & $\begin{array}{c}\text { Severe } \\
(\mathrm{CPK}>5000 \mathrm{U} / \mathrm{L}) \\
n=80\end{array}$ & $p$ Value \\
\hline Baseline eGFR $\leq 60 \mathrm{~mL} / \mathrm{min} / 1.73 \mathrm{~m}^{2}, n(\%)$ & $388(24)$ & $236(21)$ & $28(35)$ & 0.009 \\
\hline Baseline eGFR $\left(\mathrm{mL} /\right.$ minute $\left./ 1.73 \mathrm{~m}^{2}\right)$, mean $\pm \mathrm{SD}$ & $76 \pm 25$ & $77 \pm 24$ & $70 \pm 19$ & 0.01 \\
\hline Admission creatinine $(\mathrm{mg} / \mathrm{dL})$, mean $\pm \mathrm{SD}$ & $1.11 \pm 0.5$ & $1.10 \pm 0.3$ & $1.22 \pm 0.3$ & 0.05 \\
\hline Acute kidney injury, $n(\%)$ & $125(7.8)$ & $122(11)$ & $21(26)$ & $<0.001$ \\
\hline Creatinine change $(\mathrm{mg} / \mathrm{dL})$, mean $\pm \mathrm{SD}$ & $0.09 \pm 0.27$ & $0.13 \pm 0.42$ & $0.39 \pm 0.81$ & $<0.001$ \\
\hline Peak creatinine $(\mathrm{mg} / \mathrm{dL})$, mean $\pm \mathrm{SD}$ & $1.20 \pm 0.6$ & $1.23 \pm 0.6$ & $1.64 \pm 1.0$ & 0.001 \\
\hline Serum NGAL levels $(\mathrm{ng} / \mathrm{mL})$, mean $\pm \mathrm{SD}$ & $90 \pm 36$ & $112 \pm 40$ & $183 \pm 60$ & $<0.001$ \\
\hline
\end{tabular}

CPK, creatine phosphokinase; eGFR, estimated glomerular filtration rate; NGAL, neutrophil gelatinase associated lipocalin. 


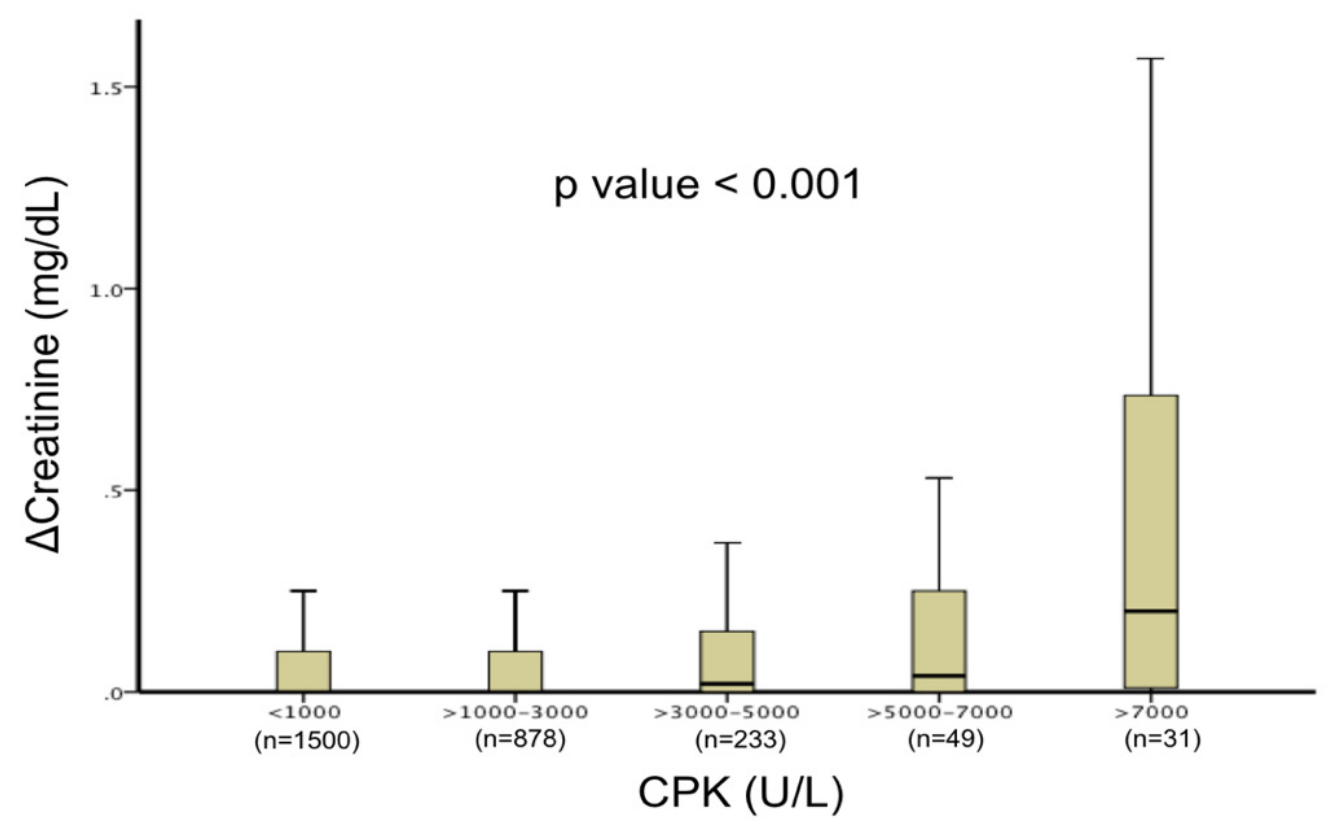

Figure 1. Relationship between CPK level elevations and creatinine changes. Box plot chart demonstrating creatinine changes stratified by peak creatine phosphokinase levels during hospitalization. A gradual increase in creatinine change was observed with rising CPK levels ( $p$ for trend $<0.001$ ).

Table 3. Multivariate binary logistic regression models predicting acute kidney injury.

\begin{tabular}{|c|c|c|c|c|}
\hline & Model 1 & \multirow{2}{*}{$p$ Value } & Model 2 & \multirow{2}{*}{$p$ Value } \\
\hline & OR $(95 \%$ CI $)$ & & OR $(95 \% \mathrm{CI})$ & \\
\hline Gender (female) & $1.2(0.8-1.8)$ & 0.5 & $1.1(0.8-1.7)$ & 0.5 \\
\hline Age (years) & $1.01(0.99-1.03)$ & 0.3 & $1.01(0.99-1.03)$ & 0.2 \\
\hline Hypertension & $1.9(1.3-2.7)$ & 0.001 & $1.8(1.3-2.7)$ & 0.001 \\
\hline LVEF (\%) & $0.93(0.92-0.95)$ & $<0.001$ & $0.93(0.91-0.95)$ & $<0.001$ \\
\hline eGFR (mL/minute/1.73 m²) & $0.97(0.96-0.98)$ & $<0.001$ & $0.97(0.96-0.98)$ & $<0.001$ \\
\hline Diabetes mellitus & $1.3(0.9-1.8)$ & 0.2 & $1.3(0.9-1.8)$ & 0.2 \\
\hline Hyperlipidemia & $1.03(0.7-1.4)$ & 0.9 & $1.07(0.8-1.5)$ & 0.7 \\
\hline Family history of CAD & $0.8(0.5-1.4)$ & 0.5 & $0.8(0.5-1.4)$ & 0.4 \\
\hline Smoking history & $0.8(0.6-1.2)$ & 0.3 & $0.8(0.6-1.2)$ & 0.3 \\
\hline Past AMI & $1.3(0.8-1.9)$ & 0.2 & $1.3(0.8-1.9)$ & 0.3 \\
\hline Peak troponin (ng/L) & $1.0(0.99-1.01)$ & 0.8 & $1.0(0.99-1.01)$ & 0.8 \\
\hline $\mathrm{CPK} \geq 1000 \mathrm{U} / \mathrm{L}$ & $1.6(1.1-2.2)$ & 0.01 & & \\
\hline $\mathrm{CPK}>5000 \mathrm{U} / \mathrm{L}^{*}$ & & & $2.8(1.4-5.6)$ & 0.004 \\
\hline
\end{tabular}

OR, odds ratio; $\mathrm{CI}$, confidence interval; $\mathrm{LV}$, left ventricular ejection fraction; eGFR, estimated glomerular filtration rate; $\mathrm{CAD}$, coronary artery disease; $\mathrm{AMI}$, acute myocardial infarction; $\mathrm{CPK}$, creatine phosphokinase. * Eighty patients with CPK > $5000 \mathrm{U} / \mathrm{L}$.

\section{Discussion}

To our knowledge, the present study is the first to describe an independent predictive value for CPK levels regarding the risk of AKI in STEMI patients, and indicates a presumable additional mechanism for kidney failure in AMI patients.

While a similar linkage is well-described for skeletal muscle breakdown [14], no study has evaluated this possible association in acute myocardial cell injury to date.

$\mathrm{CPK}$ contains dimers of $\mathrm{M}$ and $\mathrm{B}$ chains, resulting in three different forms of isoenzymes [15]. CPK-MM is generally found in high amounts in skeletal muscle cells, whereas myocardial cells consist mostly of the CK-MB fraction [4]. Although CK-MB is no longer routinely recommended for diagnosis of AMI [16], total CPK is often measured at patient admission as part of a routine chemistry blood panel. CPK is also the standard laboratory biomarker for the diagnosis and severity assessment of skeletal muscle breakdown in RM, 
and correlates strongly with AKI risk [1]. Myocardial cell destruction as in the setting of STEMI mostly causes concomitant elevation of CPK-MB as well as total CPK [5,17].

Rhabdomyolysis is accompanied by renal impairment in up to $45 \%$ of patients and AKI is considered its most serious complication $[14,18]$. Concomitant renal injury may aggravate electrolyte abnormalities and is associated with increased mortality $[3,19]$. Myoglobinuria is the cause for renal damage in this setting [20] caused by direct myoglobin toxicity to renal tubular cells, obstructive intra-tubular casts, and myoglobin-mediated renal vasoconstriction [1].

However, myoglobin has a very short half-time in serum of only a couple of minutes and is therefore seldom measured in suspected RM or myocardial infarction [21]. Furthermore, studies focusing on measuring myoglobin levels failed to establish a clear correlation to the risk of renal injury [22,23].

Elevated CPK levels were repeatedly associated with acute renal failure and the need for renal replacement therapy in patients with RM $[19,24]$. While there are no clearly defined threshold values for increased risk [14], the incidence of AKI was significantly elevated with values above $15,000[3,19]$. Nevertheless, much lower values have been proposed as well [24], especially with other factors concomitantly affecting kidney function [14,25].

AKI is a frequent complication in STEMI and is associated with increased mortality [6,7]. The worsening of renal function is multifactorial. Besides the widely known deleterious impact of contrast volume [26], other contributing factors have been shown to be involved as well. Hemodynamic changes causing impaired cardiac output, vasoactive neurohormonal activation, hypoxemia, inflammation, acidosis, hyperglycemia, and administrated nephrotoxic drugs have all been shown to correlate with worsening renal function in AMI patients [27,28].

To date, the direct impact of spillage of myocardial cell content on renal function has not been demonstrated. Although this pathogenic mechanism is well-described in skeletal muscle breakdown, with CPK serving as a sensitive independent prognostic biomarker for impending renal dysfunction, this has not yet been tested in STEMI causing cardiac muscle destruction. We observed an increased risk of kidney dysfunction even at relatively low CPK level elevations, with risk starting to rise at moderate elevations ( $>1000-5000 \mathrm{U} / \mathrm{L})$. This might be explained by the influence of the various deleterious mechanisms mentioned above, affecting the kidney simultaneously during AMI [27,28].

In the current cohort, we observed stepwise plasma NGAL level elevations among the different CPK groups. Recent data suggest the utilization of biomarkers of renal tubular damage (e.g., NGAL) in STEMI patients. These biomarkers may identify structural renal damage associated with adverse outcomes, even without functional AKI and preceding sCR elevation, thus serving as a useful tool for early AKI detection. However, they do not allow discrimination between toxic (myoglobin related) and ischemic (hemodynamic related) tubular injury $[29,30]$.

Interestingly, the presence of hypertension was associated with an increased risk of AKI. While previous data do not point to an independent direct relation between baseline hypertension and AKI, we believe that this finding in the present study may be explained by other obscure factors not included in our models associated with hypertension increasing the risk of AKI in patients receiving contrast media.

\subsection{Study Limitations}

As a single-center, retrospective, non-randomized observational study, the cohort may have been subject to bias, even though we included consecutive patients and attempted to adjust for potential confounding factors using the multivariate regression model. The risk of AKI was strongly associated with baseline eGFR and LV function. Lower eGFR and LVEF were the two strongest predictors for AKI in our study, which were stronger than peak CPK levels. Furthermore, the number of patients with CPK levels over 5000 was small. These patients had lower eGFR at baseline and lower LVEF, so it remains unclear whether the effect on kidney function was solely due to higher CPK levels. 
Data regarding the concomitant use of potentially nephrotoxic drugs such as angiotensinconverting enzyme inhibitors, mineralocorticoid receptor blockers, or diuretics was not present for many patients. Thus, we could not assess their effect on AKI development. In a similar sense, effects on CPK levels secondary to statin administration were not accessible for evaluation. Finally, changes in $\mathrm{sCr}$ can sometimes lag beyond the $48 \mathrm{~h}$ time period defined by the KDIGO criteria, and therefore a deterioration of renal function might have occurred following hospital discharge. This could have led to an underestimation of AKI incidence in our study. As a single value of eGFR does not define CKD, the true incidence of CKD could not be assessed. The definition of AKI is based on either sCr criteria or urine output criteria. We relied only on $\mathrm{sCr}$ criteria, which can be acutely elevated due to other factors, thus the diagnosis of AKI may have been overestimated. Patients with higher CPK were more likely to have lower eGFR. As information regarding patients' weight was lacking, the relation of sCr to muscle mass could not be assessed. As STEMI patients treated conservatively were not included, the true effect of PCI on AKI could not be assessed.

\subsection{Conclusions and Clinical Implications}

Total serum CPK level elevations independently predict a higher risk for AKI in STEMI patients. As opposed to the setting of rhabdomyolysis in STEMI, a significantly increased risk for acute renal dysfunction has already been observed at relatively modest CPK level elevations. Higher CPK levels suggest a higher degree of myocardial damage, which may have resulted in acutely worse LV function, and more severe adverse hemodynamic effects resulting in higher risk for AKI. In the present study, we tried differentiating whether higher CPK levels directly (myoglobin-related kidney damage) or indirectly (through hemodynamic effects) affect kidney function by adjusting the risk for AKI in the multivariate models with LVEF and troponin levels measured during hospitalization. Although presumably contributing independently, it remains unclear whether direct myoglobin-related kidney damage is the main mechanism relating CPK levels with the risk for AKI.

Our findings may bear some clinical implications. As the treatment of established AKI in STEMI patients is often limited, focus should be on identifying patients with higher risk for deterioration of renal function. Withholding nephrotoxic drugs, liberal fluid resuscitation, or alkalizing patients' urine may all be useful for patients at higher risk of AKI, identified amongst others by elevated CPK levels.

Author Contributions: Conceptualization, S.M. and D.L.; Formal analysis I.M. and S.M.; Investigation, K.-L.R.; Methodology, T.I.; Supervision, D.L. and S.B.; writing-original draft preparation, D.Z.; Writing-review and editing, S.B. and Y.S.; All authors have read and agreed to the published version of the manuscript.

Funding: This research did not receive any specific grant from funding agencies in the public, commercial, or not-for-profit sectors.

Institutional Review Board Statement: The study was conducted in accordance with the Declaration of Helsinki, and approved by the Institutional Review Board (IRB: TLV 16-224).

Informed Consent Statement: Informed consent was obtained from all subjects involved in the study.

Conflicts of Interest: The authors declare that they have no conflict of interest.

\section{References}

1. Petejova, N.; Martinek, A. Acute kidney injury due to rhabdomyolysis and renal replacement therapy: A critical review. Crit. Care Lond. Engl. 2014, 18, 224. [CrossRef] [PubMed]

2. Bagley, W.H.; Yang, H.; Shah, K.H. Rhabdomyolysis. Intern. Emerg. Med. 2007, 2, 210-218. [CrossRef] [PubMed]

3. Veenstra, J.; Smit, W.M.; Krediet, R.T.; Arisz, L. Relationship between elevated creatine phosphokinase and the clinical spectrum of rhabdomyolysis. Nephrol. Dial. Transplant. 1994, 9, 637-641. [CrossRef] [PubMed]

4. Roberts, R.; Gowda, K.S.; Ludbrook, P.A.; Sobel, B.E. Specificity of elevated serum MB creatine phosphokinase activity in the diagnosis of acute myocardial infarction. Am. J. Cardiol. 1975, 36, 433-437. [CrossRef] 
5. Yusuf, S.; Collins, R.; Lin, L.; Sterry, H.; Pearson, M.; Sleight, P. Significance of elevated MB isoenzyme with normal creatine kinase in acute myocardial infarction. Am. J. Cardiol. 1987, 59, 245-250. [CrossRef]

6. James, M.T.; Ghali, W.A.; Knudtson, M.L.; Ravani, P.; Tonelli, M.; Faris, P.; Pannu, N.; Manns, B.J.; Klarenbach, S.W.; Hemmelgarn, B.R. Alberta Provincial Project for Outcome Assessment in Coronary Heart Disease (APPROACH) Investigators, Associations between acute kidney injury and cardiovascular and renal outcomes after coronary angiography. Circulation 2011, 123, 409-416. [CrossRef]

7. Goldberg, A.; Hammerman, H.; Petcherski, S.; Zdorovyak, A.; Yalonetsky, S.; Kapeliovich, M.; Agmon, Y.; Markiewicz, W.; Aronson, D. Inhospital and 1-year mortality of patients who develop worsening renal function following acute ST-elevation myocardial infarction. Am. Heart J. 2005, 150, 330-337. [CrossRef]

8. O'gara, P.T.; Kushner, F.G.; Ascheim, D.D.; Casey, D.E.; Chung, M.K.; De Lemos, J.A.; Ettinger, S.M.; Fang, J.C.; Fesmire, F.M.; Franklin, B.A.; et al. 2013 ACCF/AHA guideline for the management of ST-elevation myocardial infarction: Executive summary: A report of the American College of Cardiology Foundation/American Heart Association Task Force on Practice Guidelines: Developed in collaboration with the American College of Emergency Physicians and Society for Cardiovascular Angiography and Interventions. Catheter. Cardiovasc. Interv. 2013, 82, E1-E27. [CrossRef]

9. Chavez, L.O.; Leon, M.; Einav, S.; Varon, J. Beyond muscle destruction: A systematic review of rhabdomyolysis for clinical practice. Crit. Care Lond. Engl. 2016, 20, 135. [CrossRef]

10. Dominguez-Rodriguez, A.; Abreu-Gonzalez, P.; Avanzas, P. Prognostic usefulness of C-reactive protein: Importance of the diurnal variation. Am. J. Cardiol. 2013, 111, 1079-1080. [CrossRef]

11. Grunau, B.; Pourvali, R.; Wiens, M.O.; Levin, A.; Li, J.; Grafstein, E.; Joo, D.; Scheuermeyer, F.X. Characteristics and thirty-day outcomes of emergency department patients with elevated creatine kinase. Acad. Emerg. Med. Off. J. Soc. Acad. Emerg. Med. 2014, 21, 631-636. [CrossRef] [PubMed]

12. LeLevey, A.S.; Stevens, L.A.; Schmid, C.H.; Zhang, Y.L.; Castro, A.F., III; Feldman, H.I.; Kusek, J.W.; Eggers, P.; Van Lente, F.; Greene, T.; et al. CKD-EPI (Chronic Kidney Disease Epidemiology Collaboration), A new equation to estimate glomerular filtration rate. Ann. Intern. Med. 2009, 150, 604-612. [CrossRef] [PubMed]

13. Stevens, P.E.; Levin, A. Kidney Disease: Improving Global Outcomes Chronic Kidney Disease Guideline Development Work Group Members, Evaluation and management of chronic kidney disease: Synopsis of the kidney disease: Improving global outcomes 2012 clinical practice guideline. Ann. Intern. Med. 2013, 158, 825-830. [CrossRef]

14. Bosch, X.; Poch, E.; Grau, J.M. Rhabdomyolysis and acute kidney injury. N. Engl. J. Med. 2009, 361, 62-72. [CrossRef]

15. Bessman, S.P.; Carpenter, C.L. The creatine-creatine phosphate energy shuttle. Annu. Rev. Biochem. 1985, 54, 831-862. [CrossRef] [PubMed]

16. Alvin, M.D.; Jaffe, A.S.; Ziegelstein, R.C.; Trost, J.C. Eliminating Creatine Kinase-Myocardial Band Testing in Suspected Acute Coronary Syndrome: A Value-Based Quality Improvement. JAMA Intern. Med. 2017, 177, 1508-1512. [CrossRef]

17. Heller, G.V.; Blaustein, A.S.; Wei, J.Y. Implications of increased myocardial isoenzyme level in the presence of normal serum creatine kinase activity. Am. J. Cardiol. 1983, 51, 24-27. [CrossRef]

18. Melli, G.; Chaudhry, V.; Cornblath, D.R. Rhabdomyolysis: An evaluation of 475 hospitalized patients. Medicine 2005, 84, 377-385 [CrossRef]

19. de Meijer, A.R.; Fikkers, B.G.; de Keijzer, M.H.; van Engelen, B.G.M.; Drenth, J.P.H. Serum creatine kinase as predictor of clinical course in rhabdomyolysis: A 5-year intensive care survey. Intensive Care Med. 2003, 29, 1121-1125. [CrossRef]

20. Zager, R.A. Studies of mechanisms and protective maneuvers in myoglobinuric acute renal injury. Lab. Investig. J. Technol. Methods Pathol. 1989, 60, 619-629.

21. Mikkelsen, T.S.; Toft, P. Prognostic value, kinetics and effect of CVVHDF on serum of the myoglobin and creatine kinase in critically ill patients with rhabdomyolysis. Acta Anaesthesiol. Scand. 2005, 49, 859-864. [CrossRef] [PubMed]

22. Beetham, R. Biochemical investigation of suspected rhabdomyolysis. Ann. Clin. Biochem. 2000, 37, 581-587. [CrossRef] [PubMed]

23. David, W.S. Myoglobinuria. Neurol. Clin. 2000, 18, 215-243. [CrossRef]

24. El-Abdellati, E.; Eyselbergs, M.; Sirimsi, H.; Van Hoof, V.; Wouters, K.; Verbrugghe, W.; Jorens, P.G. An observational study on rhabdomyolysis in the intensive care unit. Exploring its risk factors and main complication: Acute kidney injury. Ann. Intensive Care 2013, 3, 8. [CrossRef] [PubMed]

25. McMahon, G.M.; Zeng, X.; Waikar, S.S. A risk prediction score for kidney failure or mortality in rhabdomyolysis. JAMA Intern. Med. 2013, 173, 1821-1828. [CrossRef]

26. Narula, A.; Mehran, R.; Weisz, G.; Dangas, G.D.; Yu, J.; Généreux, P.; Nikolsky, E.; Brener, S.J.; Witzenbichler, B.; Guagliumi, G.; et al. Contrast-induced acute kidney injury after primary percutaneous coronary intervention: Results from the HORIZONS-AMI substudy. Eur. Heart J. 2014, 35, 1533-1540. [CrossRef]

27. Shacham, Y.; Steinvil, A.; Arbel, Y. Acute kidney injury among ST elevation myocardial infarction patients treated by primary percutaneous coronary intervention: A multifactorial entity. J. Nephrol. 2016, 29, 169-174. [CrossRef]

28. Marenzi, G.; Cosentino, N.; Bartorelli, A.L. Acute kidney injury in patients with acute coronary syndromes. Heart Br. Card. Soc. 2015, 101, 1778-1785. [CrossRef] 
29. Merdler, I.; Rozenfeld, K.-L.; Zahler, D.; Shtark, M.; Goldiner, I.; Loewenstein, I.S.; Fortis, L.; Hochstadt, A.; Keren, G.; Banai, S.; et al. Neutrophil Gelatinase-Associated Lipocalin for the Early Prediction of Acute Kidney Injury in ST-Segment Elevation Myocardial Infarction Patients Treated with Primary Percutaneous Coronary Intervention. Cardiorenal Med. 2020, 10, 154-161. [CrossRef]

30. Rozenfeld, K.-L.; Zahler, D.; Shtark, M.; Goldiner, I.; Keren, G.; Banai, S.; Shacham, Y. Elevated Neutrophil Gelatinase-Associated Lipocalin for the Assessment of Structural versus Functional Renal Damage among ST-Segment Elevation Myocardial Infarction Patients. Blood Purif. 2020, 49, 560-566. [CrossRef] 